\title{
Implementation of a Key Exchange Protocol Using Real Quadratic Fields
}

\section{Extended Abstract}

\author{
Renate Scheidler \\ Department of Computer Science \\ University of Manitoba \\ Winnipeg, Manitoba \\ Canada R3T 2N2 \\ Johannes A. Buchmann \\ FB-10 Informatik \\ Universität des Saarlandes \\ 6600 Saarbrücken \\ West Germany \\ Hugh C. Williams \\ Department of Computer Science \\ University of Manitoba \\ Winnipeg, Manitoba \\ Canada R3T 2N2
}




\title{
Implementation of a Key Exchange Protocol Using Real Quadratic Fields
}

\author{
Extended Abstract
}

\section{Introduction}

In [1] Buchmann and Williams introduced a key exchange protocol which is based on the Diffie-Hellman protocol (see [2]). However, instead of employing arithmetic in the multiplicative group $F^{*}$ of a finite field $F$ (or any finite Abelian group $G$ ), it uses a finite subset of an infinite Abelian group which itself is not a subgroup, namely the set of reduced principal ideals in a real quadratic field. As the authors presented the scheme and its security without analyzing its actual implementation, we will here discuss the algorithms required for implementing the protocol.

Let $D \in \mathrm{Z}_{+}$be a squarefree integer, $K=\mathrm{Q}+\mathrm{Q} \sqrt{D}$ the real quadratic number field generated by $\sqrt{D}$, and $\mathbf{O}=\mathrm{Z}+\mathrm{Z} \frac{\sigma-1+\sqrt{D}}{\sigma}$ the maximal real quadratic order in $K$, where $\sigma=\left\{\begin{array}{lll}1 & \text { if } D \equiv 2,3 \quad(\bmod 4) \\ 2 & \text { if } D \equiv 1 & (\bmod 4)\end{array}\right.$.

A subset $\mathbf{a}$ of $\mathrm{O}$ is called an ideal in $\mathrm{O}$ if both $\mathbf{a}+\mathbf{a}$ and $\mathbf{O} \cdot \mathbf{a}$ are subsets of $\mathbf{a}$. An ideal is said to be primitive if it has no rational prime divisors. Each primitive ideal $\mathbf{a}$ in $\mathbf{O}$ has a representation

$$
\mathbf{a}=\left[\frac{Q}{\sigma}, \frac{P+\sqrt{D}}{\sigma}\right]=\mathbf{Z} \frac{Q}{\sigma}+\mathbf{Z} \frac{P+\sqrt{D}}{\sigma}
$$


where $P, Q \in \mathrm{Z}, Q$ is a divisor of $D-P^{2}$ (see [5]). Let $\Delta=\frac{4}{\sigma^{2}} D$ denote the discriminant of $K$, set $d=\lfloor\sqrt{D}\rfloor$.

A principal ideal $\mathbf{a}$ of $\mathrm{O}$ is an ideal of the form $\mathbf{a}=\frac{1}{\alpha} \mathbf{O}, \alpha \in K-[0\}$. Denote by $\mathbf{P}$ the set of primitive principal ideals in $\mathrm{O}$. An ideal $\mathrm{a}=\frac{1}{\alpha} \mathrm{O} \in \mathrm{P}$ is reduced if and only if $\alpha$ is a minimum in 0 , i.e. if $\alpha>0$ and there exists no $\beta \in 0-\{0\}$ such that $|\beta|<\alpha$ and $\left|\beta^{\prime}\right|<\alpha$. Since the set $\{\log \alpha \mid \alpha$ is a minimum in $O$ \} is discrete in the real numbers $R$, the minima in $\mathbf{O}$ can be arranged in a sequence $\left(\alpha_{j}\right) j \in Z$ such that $\alpha_{j}<\alpha_{j+1}$ for all $j \in \mathbf{Z}$. If we define $a_{j}=\frac{1}{\alpha_{j}} O$ for all $j \in Z$, then the set $\Re$ consisting of all reduced ideals in $P$ is finite and can be written as $\Re=\left\{\mathbf{a}_{1}, \ldots, \mathbf{a}_{l}\right\}$ where $l \in \mathbf{Z}_{+}$.

Define an (exponential) distance between two ideals $\mathbf{a}, \mathbf{b} \in \mathfrak{R}$ as follows:

$\lambda(\mathbf{a}, \mathbf{b})=\alpha$ where $\alpha \in K^{>0}$ is such that $\mathbf{b}=\frac{1}{\alpha} \mathbf{a}$ and $\log \alpha$ is minimal.

(The logarithm of this distance function is exactly the distance as defined in [1] and [4].) Similarly, let the distance between an ideal $\mathrm{a} \in \mathfrak{N}$ and a positive real number $x$ be $\lambda(\mathbf{a}, x)=\frac{e^{x}}{\alpha}$ where $\alpha \in K^{>0}$ is such that $\mathrm{a}=\frac{1}{\alpha} \mathrm{O}$ and $\mid x-\log \alpha \mathrm{l}$ is minimal.

Throughout our protocol the inequalities $\eta^{-\frac{1}{4}}<\lambda(a, b), \lambda(a, x)<\eta^{\frac{1}{4}}$ will be satisfied for all $\mathbf{a}, \mathbf{b} \in \mathfrak{R}, x \in \mathbf{R}_{+}$, where $\eta$ is the fundamental unit of $K$.

Lemma 1: Let $\mathbf{b} \in \Re$ and write $\mathbf{b}=\mathbf{b}_{j}, \mathbf{b}_{k}=\left[\frac{Q_{k-1}}{\sigma}, \frac{P_{k-1}+\sqrt{D}}{\sigma}\right]$ for $k \geq j$. Then the following is true:

a) $\quad \mathbf{b}_{k} \in \mathfrak{R}$ and $0<P_{k} \leq d, 0<Q_{k} \leq 2 d$ for $k \geq j$, 
b) $1+\frac{1}{\sqrt{\Delta}}<\lambda\left(\mathbf{b}_{j+1}, \mathbf{b}_{j}\right)<\sqrt{\Delta}$

c) $\lambda\left(\mathbf{b}_{j+2}, \mathbf{b}_{j}\right)>2$,

d) If $\mathbf{b}=\frac{1}{\beta} \mathbf{0}, \beta \in K_{>0}$, then $\lambda(\mathbf{b}, x)=\frac{e^{x}}{\beta}$,

e) $\lambda\left(\mathbf{b}_{k}, \mathbf{b}_{j}\right)=\frac{\lambda\left(\mathbf{b}_{k}, x\right)}{\lambda\left(\mathbf{b}_{j}, x\right)}$ for any $x \in \mathbf{R}_{+}, k \geq j$.

Since principal ideal generators and distances are generally irrational numbers, we need to use approximations in our protocol. Denote by $\mathbf{a}(x)$ the reduced ideal closest to $x \in \mathbf{R}_{+}$, i.e. $|\log \lambda(\mathbf{a}(x), x)|<|\log \lambda(\mathbf{b}, x)|$ for any $\mathbf{b} \in \Re, \mathbf{b} \neq \mathbf{a}$, and by $\hat{\mathrm{a}}(x)$ the ideal actually computed by our algorithm. Define $a_{+}(x)$ to be the reduced ideal such that its distance to $x$ is maximal and $<1$. Similarly, $\lambda\left(\mathbf{a}_{-}(x), x\right)>1$ and minimal. Let $\lambda_{1}(x)=\lambda(\mathbf{a}(x), x)$, $\lambda_{2}(x)=\lambda(\hat{a}(x), x)$. Denote by $\hat{\lambda}(\mathbf{a}, x)$ the approximation of $\lambda(\mathrm{a}, x)$ computed by our algorithm; write $\hat{\lambda}(\mathbf{a}, x)=\frac{M(\mathbf{a}, x)}{2^{p}}$ where $M(\mathbf{a}, x) \in \mathbf{Z}_{+}$and $p \in \mathbf{Z}_{+}$is a precision constant to be determined later. $\hat{\lambda}_{1}(x), \mathrm{M}_{1}(x), \hat{\lambda}_{2}(x), \mathrm{M}_{2}(x)$ are defined analogously to $\hat{\lambda}(x)$ and $\mathrm{M}(x)$ with respect to $\lambda_{1}(x)$ and $\lambda_{2}(x)$. Set

$$
G=1+\frac{1}{15(d+1)}, \quad \gamma=\left\lceil G^{-1} 2^{p}\right\rceil, \quad \chi=1+\frac{1}{2^{p-1}} .
$$

The protocol can be outlined as follows: Two communication partners $A$ and $B$ agree publicly on a small number $c \in \mathbf{R}_{+}$and an initial ideal $\mathrm{A}(c)$ with approximate distance $\mathrm{M}_{2}(c)$ from $c$. A secretly chooses $a \in\{1, \ldots, d\}$, computes $\hat{\mathrm{A}}(a c)$ and $\mathrm{M}_{2}(a c)$ from $\hat{\mathrm{A}}(c)$ and $\mathrm{M}_{2}(c)$, and sends both to B. Similarly, B secretly chooses $b \in\{1, \ldots, d\}$, calculates $\mathrm{A}(b c)$ and $\mathrm{M}_{2}(b c)$, and transmits both to $A$. Now both communication partners are able to determine an ideal $\mathrm{A}(a b c)$. Although this ideal need not be the same for $\mathrm{A}$ and $\mathrm{B}$ (due to 
their different approximation errors in the computation), a little additional work will enable them to agree on a common ideal which is the secret key.

As pointed out in [1], we expect $l=|\Re| \gg D^{\frac{1}{2}-\varepsilon}$ for arbitrary $\varepsilon$ if $D$ is chosen correctly and sufficiently large. This shows that an exhaustive search attack is infeasible. The authors conjecture that breaking the protocol enables one to factor. In [1] it is proved that solving the discrete logarithm problem for reduced principal ideals in real quadratic orders given $\mathbf{a} \in \mathscr{K}$ find $\lambda(\mathbf{a}, x)$ - in polynomial time implies being able to both break the scheme and factor $D$ in polynomial time.

Throughout the protocol we will assume $M(\mathbf{a}, x) \geq \gamma$ for all $\mathbf{a} \in \Re$ and $x \in \mathbf{R}_{+}$. Any number $\theta \in K$ is approximated by $\hat{\theta} \in \mathrm{Q}$ such that $\chi^{-1} \theta \leq \hat{\theta} \leq \chi \theta$.

\section{The Algorithms}

For our protocol we need to perform arithmetic in both $\mathbf{P}$ and $\mathfrak{R}$. Our first algorithm enables us to compute any reduced ideal $\mathbf{a}_{k}$ from a given reduced ideal $\mathbf{a}_{j}$ by simply going through $\Re$ "step by step".

Algorithm 1 (Neighbouring in $\Re$ ): Input: $\mathbf{a}_{j} \in \mathfrak{K}$.

Output: The neighbours $\mathbf{a}_{j+1}, \mathbf{a}_{j-1} \in \Re$ and $\psi_{+}, \psi_{-}$such that $\mathbf{a}_{j \pm 1}=\psi_{ \pm} \mathbf{a}_{j}$.

Algorithm: $\mathbf{a}_{j+1}$ is obtained by computing one iteration in the continued fraction expansion of the irrational number $\frac{P_{j-1}+\sqrt{D}}{Q_{j-1}}$. The algorithm for $a_{j-1}$ is the inverse of the algorithm for $a_{j+1}$. In particular: 


$$
\begin{aligned}
& q_{j-1}=\left\lfloor\frac{P_{j-1}+d}{Q_{j-1}}\right\rfloor, \quad P_{j}=q_{j-1} Q_{j-1}-P_{j-1}, \quad Q_{j}=\frac{D-P_{j}^{2}}{Q_{j-1}}, \quad \psi_{+}=\frac{\sqrt{D}-P_{j}}{Q_{j}}, \\
& Q_{j-2}=\frac{D-P_{j-1}^{2}}{Q_{j-1}}, \quad q_{j-2}=\left\lfloor\frac{P_{j-1}+d}{Q_{j-2}}\right\rfloor, P_{j-2}=q_{j-2} Q_{j-2}-P_{j-1}, \quad \Psi_{-}=\frac{\sqrt{D}+P_{j-1}}{Q_{j-2}} .
\end{aligned}
$$

Algorithm 2 (Multiplication in P): Input: a, $\mathbf{a}^{\prime} \in \mathrm{P}$.

Output: $U \in \mathbf{Z}_{\geq 0}, \mathbf{c} \in \mathbf{P}$ such that $\mathbf{a a}^{\prime}=U \mathbf{c}$.

Algorithm: See [3], [4].

Lemma 2: If $\mathbf{a}=\mathbf{a}_{s}, \mathbf{a}=\mathbf{a}_{t}$ such that $\mathbf{a}_{s-1}, \mathbf{a}_{t-1} \in \mathfrak{K}$, then Algorithm 2 performs $\mathrm{O}(\log D)$ arithmetic operations on numbers of input size $\mathrm{O}(\log D)$.

Proof: By Lemma 1 all input numbers are polynomially bounded in $D$. The algorithm performs a fixed number of arithmetic operations plus two applications of the Extended Euclidean Algorithm which has complexity $O(\log D)$.

Algorithm 3 (Reduction in P): Input: $\mathbf{c}=\left[\frac{Q}{\sigma}, \frac{P+\sqrt{D}}{\sigma}\right] \in \mathbf{P}$.

Output: $\mathbf{b} \in \Re, G, B \in \mathbf{Z}_{\geq 0}$ such that $\theta=\frac{G+B \sqrt{D}}{Q}$ and $\mathbf{b}=\theta \mathbf{c}$.

Algorithm: The algorithm is very similar to Algorithm 1 and uses again the continued fraction expansion of $\frac{P+\sqrt{D}}{Q}$ (see [3]). 
Lemma 3: If $\mathbf{c}=\frac{1}{U} \mathbf{a}_{s} \mathbf{a}_{t}$ where $\mathbf{a}_{s}, \mathbf{a}_{t}$ are as in Lemma 2, then Algorithm 3 performs $\mathrm{O}(\log D)$ arithmetic operations on numbers of input size $\mathrm{O}(\log D)$.

Proof: By [5], Algorithm 2, and Lemma 1, the maximun number of iterations is $\mathrm{O}(\log D)$. The bound on the input size follows from Lemma 1 and results in [4].

Algorithm 4: Input: $\hat{\mathbf{a}}(x), \hat{\mathrm{a}}(y) \in \Re, \mathrm{M}_{2}(x), \mathrm{M}_{2}(y)$ for $x, y \in \mathbf{R}_{+}$. Output: $\hat{\mathbf{a}}(x+y) \in \Re, \mathrm{M}_{2}(x+y)$.

Algorithm: First use Algorithm 2 to compute $U \in \mathrm{Z}, \mathrm{c}=\left[\frac{Q}{\sigma}, \frac{P+\sqrt{D}}{\sigma}\right] \in \mathbf{P}$ such that $(U) \mathbf{c}=\hat{\mathbf{a}}(x) \hat{\mathbf{a}}(y)$. Then compute $\mathbf{b}=\left[\frac{Q^{\prime}}{\sigma}, \frac{P^{\prime}+\sqrt{D}}{\sigma}\right] \in \Re$ and $G, B \in \mathbf{Z}_{\geq 0}$ such that $\mathbf{b}=\theta \mathbf{c}, \theta=\frac{G+B \sqrt{D}}{Q}$ using Algorithm 3. Finally apply Algorithm 1 to $\mathbf{b}$ a certain number of times to obtain $\hat{\mathbf{a}}(x+y)=\zeta \mathbf{b}=\frac{\zeta \theta}{U} \hat{\mathrm{a}}(x) \hat{\mathrm{a}}(y)$. Set

$$
\mathrm{M}_{2}(x+y)=\left\lceil\frac{\zeta \hat{\theta} \mathrm{M}_{2}(x) \mathrm{M}_{2}(y)}{2 p U}\right\rceil \text {. }
$$

where $\hat{\zeta}, \hat{\theta}$ are rational approximations to $\zeta, \theta$, respectively.

Lemma 4: If $\hat{\mathrm{a}}(x)=\mathrm{a}_{s}, \hat{\mathrm{a}}(y)=\mathrm{a}_{t}$ such that $\mathrm{a}_{s-1}, \mathrm{a}_{t-1} \in \Re$, then Algorithm 4 performs $\mathrm{O}(\log D)$ arithmetic operations on inputs of size $\mathrm{O}(\log D)$.

Proof:By Lemma 2, computing $\mathbf{c}$ takes $\mathrm{O}(\log D)$ arithmetic operations on inputs of size $\mathrm{O}(\log D)$. By Lemma 3, the same is true for the computation of $\mathbf{b}$. From Lemma 1 it can be proved that, in obtaining $\mathrm{a}(x+y)$ from $b$, all numbers involved are polynomially bounded in $D$ and $\mathrm{A}(x+y)$ can be obtained from $\mathbf{b}$ in $\mathrm{O}(\log D)$ iterations. 
Both communication partners can determine the key by using the following algorithm which is based on the idea of a standard exponentiation method:

Algorithm 5: Input: $\hat{\mathrm{a}}(x) \in \Re$ for $x \in \mathbf{R}_{+}, \mathrm{M}_{2}(x), y \in \mathrm{Z}_{+}$. Output: $\hat{\mathrm{a}}(x y), \mathrm{M}_{2}(x y)$.

Algorithm: 1) Determine the binary decomposition $y=\sum_{i=0}^{l} b_{i} 2^{l-i}$ of $y, b_{i} \in\{0,1\}, b_{0}=1$.

2) $\operatorname{Set} \hat{\mathrm{A}}\left(z_{0}\right)=\mathrm{A}(x)$.

3) for $i=1$ to $l \mathrm{do}$

a) Compute $\mathrm{A}\left(2 z_{i-1}\right), \mathrm{M}_{2}\left(2 z_{i-1}\right)$ using Algorithm 4 .

$$
\text { Set } \hat{A}\left(z_{i}\right):=\mathrm{A}\left(2 z_{i-1}\right), \mathrm{M}_{2}\left(z_{i}\right):=\mathrm{M}_{2}\left(2 z_{i-1}\right) \text {. }
$$

b) if $b_{i}=1$ then compute $\mathrm{a}\left(z_{i}+x\right), \mathrm{M}_{2}\left(z_{i}+x\right)$ using Algorithm 4 .

$$
\text { Set } \hat{\mathrm{A}}\left(z_{i}\right):=\mathrm{A}\left(z_{i}+x\right), \mathrm{M}_{2}\left(z_{i}\right):=\mathrm{M}_{2}\left(z_{i}+x\right) \text {. }
$$

4) Set $\mathrm{A}(x y):=\mathrm{A}\left(z_{l}\right), \mathrm{M}_{2}(x y)=\mathrm{M}_{2}\left(z_{l}\right)$.

Lemma 5 : If $\hat{\mathrm{a}}(x)=\mathrm{a}_{s}$ such that $\mathrm{a}_{s-1} \in \Re$ and $y$ is polynomially bounded in $D$, then Algorithm 5 performs $\mathrm{O}\left((\log D)^{2}\right)$ arithmetic operations on inputs of size $\mathrm{O}(\log D)$.

Proof: For each iteration, steps $3 a$ and $3 b$ each perform $O(\log D)$ operations on numbers of input size $O(\log D)$ by Lemma 4 . So the number of operations needed for step 3 is $\mathrm{O}(l \log D)=\mathrm{O}\left((\log D)^{2}\right)$ 


\section{The Protocol}

Algorithm 6 (Initial values): Input: $r \in\{2, \ldots, d\}$.

Output: $\mathbf{a} \in \mathfrak{R}, \mathbf{M} \in \mathbf{Z}_{+}$, such that the ideal $\mathbf{a}$ and its distance $M$ can be used as initial values for the protocol.

Algorithm: Set $\mathbf{a}=\hat{\mathrm{a}}(c)=\mathbf{0}, \mathrm{M}=\mathrm{M}_{2}(c)=\left\lceil 2^{p} r\right\rceil$, where $c=\log r$. Then $\mathrm{M} \geq 2^{p+1}>\gamma$. Since $1+\frac{1}{\sqrt{\Delta}}<r=\lambda_{2}(c)<\sqrt{\Delta}$, we have $\mathbf{a}=\mathbf{a}_{-}(c)$.

In order to find a unique key ideal, all approximation errors $\rho_{2}(x)=\frac{\hat{\lambda}_{2}(x)}{\lambda_{2}(x)}\left(x \in \mathbf{R}_{+}\right)$in Algorithms 4,5 , and 6 must be close to 1 , i. e. $p$ must be sufficiently large.

Theorem 1: Let $a, b \in\{1, \ldots, d\}, \hat{\mathrm{a}}(c), \mathrm{M}_{2}(c)$ as in Algorithm 6. Let $\mathrm{A}(a b c)$ be computed by applying Algorithm 5 first to $\hat{\mathrm{a}}(c), \mathrm{M}_{2}(c)$, and $b$ to obtain $\hat{\mathrm{a}}(b c)$ and $\mathrm{M}_{2}(b c)$, then to $\hat{\mathrm{a}}(b c), \mathrm{M}_{2}(b c)$, and $a$ to obtain $\hat{\mathrm{a}}(a b c)$ and $\mathrm{M}_{2}(a b c)$. If $2 p \geq 1280 d\left(d^{2}-1\right)$, then $\hat{\mathrm{A}}(a b c) \in\left\{\mathbf{a}_{-}(a b c), \mathbf{a}_{+}(a b c)\right\}$ and $\mathrm{M}_{2}(a b c) \geq \gamma$.

The uniqueness of the key ideal is guaranteed by the following Lemma:

Lemma 6: Let $p, a, b, c, \hat{\mathrm{A}}(c), \mathrm{M}_{2}(c)$ be as in Theorem 1 . Set $x=a b c$.

If $\lambda_{1}(x)>G^{2}$ or $\lambda_{1}(x)<G^{-2}$ then $\mathrm{A}(x)=\mathrm{a}_{-}(x)$.

If $G^{-2} \leq \lambda_{1}(x) \leq G^{2}$ then $\mathbf{a}(x)$ can be determined from $\mathrm{a}(x)$.

Proof: Omit the argument $x$ for brevity. If $\lambda_{1}>G^{2}$ or $\lambda_{1}<G^{-2}$ then $\hat{\lambda}_{2}>G$ and hence $\lambda_{2}=\frac{\hat{\lambda}_{2}}{\rho_{2}}>1$, so $\hat{\mathbf{a}}=\mathbf{a}$.. 
If $G^{-2} \leq \lambda_{1} \leq G^{2}$, then by Theorem $1 \mathbf{a} \in\left\{\mathbf{a}_{+}, \mathbf{a}_{-}\right\}$, so $\mathbf{a}=\mathbf{a}$ or $\mathbf{a}$ is one of the neighbours of $\hat{a}$. From Theorem 1 it can be proved that $G^{-1} \leq \rho_{2} \leq G$ and hence $G^{-3} \leq \hat{\lambda}_{1}<\frac{1+2-\mathrm{P}}{1-G^{3} 2 \mathrm{P}} G^{3}$. So both communication partners can determine an ideal $\mathbf{b}$ which is either $\hat{\mathbf{a}}$ or a neighbour of $\hat{\mathfrak{a}}$ such that $G^{-3} \leq \hat{\lambda}(\mathbf{b}, a b c)<\frac{1+2-p}{1-G^{3} 2^{2}} G^{3}$. Then it can be shown that $\frac{1}{1+\frac{1}{\sqrt{\Delta}}}<\lambda(\hat{a}, \mathbf{b})<1+\frac{1}{\sqrt{\Delta}}$ therefore by Lemma $1: \hat{\mathbf{a}}=\mathbf{a}$.

We are now equipped to set up the protocol. We assume $2 p \geq 1280 d\left(d^{2}-1\right)$.

\section{Protocol:}

The two communication partners Alice and Bob perform the following steps:

1) Both Alice and Bob agree on $D$ and a small positive integer $r$. They compute $\mathbf{a}=\hat{\mathbf{a}}(c)$, $\mathrm{M}=\mathrm{M}_{2}(c) \geq \gamma$ using Algorithm 6 where $c=\log r . D, \mathbf{a}$, and $\mathrm{M}$ can be made public.

2) Alice secretly chooses $a \in\{1, \ldots, d\}$ and from $\mathrm{a}, \mathrm{M}$ computes $\hat{\mathrm{a}}(a c), \mathrm{M}_{2}(a c) \geq \gamma$ using Algorithm 5. She sends both to Bob.

3) Bob secretly chooses $b \in\{1, \ldots, d\}$ and from $a, M$ computes $\mathrm{a}(b c), \mathrm{M}_{2}(b c) \geq \gamma$ using Algorithm 5. He sends both to Alice.

4) From $\mathrm{A}(a c), \mathrm{M}_{2}(a c)$, and $b$, Bob computes $\mathrm{A}(a b c)$ and its two neighbours as well as their approximate distances (i.e. $M$ values) using Algorithms 5 and 1 . If he finds among these an ideal $\mathbf{b}$ such that $\frac{2 p}{G^{3}} \leq \mathrm{M}(\mathbf{b}, a b c)<\frac{(1+2 p) G^{3}}{1-2 p G^{3}}$, then $\mathbf{b}=\mathbf{a}(a b c)$. In this case he sends 
' 0 ' back to Alice. If he cannot find such an ideal, then by Lemma 6 he can compute $a_{-}(a b c)$. In this case he sends ' 1 ' to Alice.

5) From $\mathrm{A}(b c), \mathrm{M}_{2}(b c)$, and $a$, Alice computes $\hat{\mathrm{a}}(a b c), \mathrm{M}_{2}(a b c)$ using Algorithm 5 . If she received ' 0 ' from Bob, then she computes the neighbours of $\mathrm{A}(a b c)$ and their $M$ values and attempts to compute $\mathbf{a}(a b c)$. If successful, she sends ' 0 ' back to Bob. The common key is then $\mathbf{a}(a b c)$. Otherwise the ideal $\hat{\mathrm{a}}(a b c)$ she computed is $\mathrm{a}_{-}(a b c)$. In this case she sends ' 1 ' to Bob. If Alice received ' 1 ' from Bob, then he was unable to determine $\mathbf{a}(a b c)$, so we must have $\lambda_{1}(a b c)<G^{-2}$ or $\lambda_{1}(a b c)>G^{2}$ by Lemma 6 , in which case the ideal $a(a b c)$ computed by Alice is $a_{-}(a b c)$. This is then the key. In this case she sends ' 1 ' back to Bob.

6) If Bob receives the same bit he sent, then the ideal he computed in step 4 is the key. The only other possibility is that he sent ' 0 ' and received ' 1 '. In this case Alice was unable to determine $\mathbf{a}(a b c)$. The key is then the ideal $\hat{\mathrm{a}}(a b c)=\mathbf{a}_{-}(a b c)$ initially computed by Bob.

\section{References:}

[1] J. A. Buchmann, H. C. Williams, A key exchange system based on real quadratic fields, extended abstract, to appear in: Proceedings of CRYPTO '89.

[2] W. Diffie, M. Hellman, New directions in cryptography, IEEE Trans. Inform. Theory, vol. 22, 1976.

[3] R. A. Mollin, H. C. Williams, Computation of the class number of a real quadratic field, to appear in: Advances in the Theory of Computation and Computational Mathematics (1987).

[4] A. J. Stephens, H. C. Williams, Some computational results on a problem concerning powerful numbers, Math. of Comp. vol. 50, no. 182, April 1988. 
[5] H. C. Williams, M. C. Wunderlich, On the parallel generation of the residues for the continued fraction factoring algorithm, Math. of Comp. vol. 48, no. 177, January 1987. 